\title{
A CREACIÓN DE ESPACIOS PARA REFLEXIONAR Y MEJORAR EL CAMPO DE LA CERÁMICA
}

Denisse Estefania Gracia Alcázar Universidad Nacional De Colombia 


\begin{abstract}
El Taller de Cerámica de la Universidad Nacional de Cerámica, se ha pensado como un lugar de experimentación colectiva e individual. De investigación creación, en donde el estudiante puede conocer y vivenciar el lenguaje cerámico a partir del conocimiento de sus conceptos, su historia, sus técnicas, la práctica y experimentación de estas. Es allí donde se busca apropiar los procesos de los materiales cerámicos para posteriormente articularlos al desarrollo de los trabajos personales y exploración de cada estudiante. Esta búsqueda dentro de los procesos de investigación creación de cada persona. Fortalece y nutre su mirada al encontrar en la materia cerámica nuevas formas de pensar y percibir, propias del hacer. Este texto habla de las iniciativas y actividades que se han desarrollado en pro de estos lineamientos.
\end{abstract}

Palabras-Claves: Cerámica-artística. Investigación-Creación. Educación-Artística.

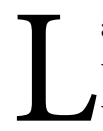
a cerámica ha sido un lenguaje plástico, que en Colombia, ha tenido un gran estigma. Desde hace varios años, este hacer es visto como un oficio menor, enfocado principalmente a lo utilitario olvidando o dejando de lado todo su potencial plástico y artístico. Inscrita a la categoría de oficio menor, durante muchos años ha sido vista como una artesanía. Su función tradicional, utilitaria y decorativa la ha alejado de los pensum de las artes y de las instituciones artísticas. "Esta jerarquización de las practicas creativas no existió siempre. La diferencia de status entre los -oficios de las artes- (artesanía) y las -artes plásticas-, entre el artesano y el artista, se estableció más o menos en el Renacimiento y se institucionalizó progresivamente con el desarrollo socio-económico de los últimos siglos. El siglo 20 conoció un cambio muy grande de las prácticas artísticas sin que se hubiera cuestionado esta jerarquía entre el arte mayor y el arte menor" (Art Press 2, 2013, pág. 29).

A diferencia de otros países, sus múltiples posibilidades artísticas no han sido muy conocidas y están todavía por explorar y conocer en nuestro país. En Colombia hemos tenido algunos intentos por visibilizar la cerámica pero aun hoy esta mirada sesgada, que hemos heredada, no nos ha permitido verla diferente. Los prejuicios culturales heredados nos han enseñado que la porcelana y la loza fina, proveniente de Europa, eran mejores, más finas, sublime y delicadas, negando por otro lado toda la tradición cerámica precolombina existente en nuestro territorio. Sabemos que para los precolombinos la cerámica cumplía un papel fundamental y era a través de estos que transmitan sus costumbres, desarrollaban instrumentos, objetos rituales y de su cotidianidad. Su desarrollo técnico respondía al conocimiento del material y sus necesidades. Con la llegada de los españoles se da un giro a los valores y a partir del periodo colonial 
se comienzan a priorizas los ideales europeos. Este cambio de mirada da lugar a una perspectiva euro-centrista, sobrevalorándola dejando de lado lo local y propio. Incluso desde el campo del arte Marta Traba en el último capítulo de su Historia Abierta del Arte Colombiano se refiere a la cerámica que se produjo en nuestro país como torpe y sin mayores atributos “(...) nada revela más claramente el extraordinario talento de las culturas mejicanas frente a las culturas peruanas (...) y frente a la pobreza, a la limitación determinada por la utilidad y la repetición de moldes, de la cerámica colombiana y de la ecuatoriana" (Traba, 1985). Sentando un precedente sobre la falta de investigación en el campo de la cerámica precolombina desde la creación artística en ese momento, los prejuicios heredados que se seguían heredando y la constante comparación y punto de referencia con Europa.

Como egresada de la Escuela de Artes Plásticas de la UN (20032009), el interés por la cerámica estuvo presente desde los primeros semestres de formación. En la búsqueda plástica por un lenguaje afín a los interese artísticos encontré la cerámica, y ella sus posibilidades matericas, escultóricas, sonoras, constructivas, graficas, objetuales e instalativas. Sin embargo el panorama de la cerámica no era muy alentador y el taller de cerámica de la Universidad Nacional había sido dejado al olvido académico generando así que se pensara varias veces en cerrarlo. Esto no se hacía, gracias a las pocas actividades desarrolladas como cursos académicos, proyectos estudiantiles y eventos extracurriculares que mostraban que el espacio todavía era habitado.

Fue debido a esto, que en el 2009 y como parte del trabajo final de la formación como artista, se realizó una investigación que buscaba entender el estado del campo de la cerámica y sus problemáticas (Gracia, Volumen 2 Reflexiones, 2009). A partir de entrevistas realizadas a ceramistas, historiadores y docentes de artes relacionados con la Cerámica se pudo comprender que estaba sucediendo. Ceramistas- escultores como Nijole Siviskas, Tina Vallejo, Carol Young, Cecilia Ordoñez, Diana Lamir, Augusto Rocha, Marcela Woolcott, Martha Combariza, Nubia Roncancio, Juanita Richter, Any Sepulveda, Jorge Pérez, Jorge Pachón, el historiador German Rubiano y los docentes María Teresa Pardo y David Lozano, entre otros entrevistados, aportaron sus miradas, vivencias y experiencia en esta área.

La problemática que estos veían en el campo de la cerámica eran:

- Por varios años se había visto un campo de la cerámica atomizado y desarticulado en donde el conocimiento no circula libremente.

- Los espacios de formación tanto formales como informales de aprendizaje de la cerámica eran muy pocos, casi nulos, obligando a los interesados a ser autodidactas o a partir al exterior para ampliar sus 
conocimientos.

- La inexistencia de escuelas de aprendizaje sobre la cerámica vista como profesión o como un proceso de investigación-creación y exploración diversas a lo utilitario hacían que no existiera el ciclo completo en el campo.

- Esto generaba a su vez que faltaran materiales y proveedores de herramientas, de equipos que facilitasen este hacer y su diversificación.

Esta investigación ayudo a comprender como estaba el campo de la cerámica y a entender cuáles eran las necesidades puntuales y falencias del mismo para poder crear actividades y acciones basándose en los puntos en los que se debían reforzar.

Adicional a esta investigación, fue la experiencia de formación profesional como ceramista en Francia y el desarrollo de maestría en Prácticas Artísticas y Acción Social en donde se tuvo la posibilidad de indagar sobre el campo de la cerámica en Francia. Estos estudios permitieron descubrir, entender y aprender sobre aspecto de la cerámica vista como Arte.

Las herramientas conceptuales, técnicas, prácticas adquiridas, entre otras, posibilitaron comprender y entender las necesidades que tenía el espacio y la cerámica como campo pensándose como un conocimiento global y una forma de trasmitir y motivar a partir de su enseñanza y sus requerimientos. Haber visto otras escuelas y tipos de enseñanza me permitía tener una mirada clara sobre lo que se podía plantear. Un aprendizaje que no podría separar el hacer, del pensar y que vinculaba el concepto, la historia, las técnicas, la práctica, la investigación-exploración y la creación.

\section{¿Como?}

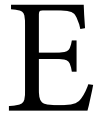

nseñar la cerámica no sólo implica dar a conocer la técnica. "El valor de la educación artística reside en su facultad de aproximarnos al mundo y sus gentes, porque nos enseña tangiblemente lo que otros piensan, a comprender, admitir y tolerar otras formas de sentir y de pensar" (Garcia, 2017). Se debe enseñar la cerámica desde sus infinitas posibilidades y no desde sus prejuicios. Enseñarlo desde la pregunta que tiene cada estudiante y que a través del trabajo y conocimiento sensible de los procesos y la exploración logra materializar. Ensañar la cerámica, es enseñar un lenguaje con múltiples caminos por recorrer como las de la pintura, el dibujo, la fotografía, el grabado, la escultura entre otras y que a la vez la cerámica puede volverse todo ello; puede ser dibujo, puede ser escultura, puede ser grabado, puede ser fotografía, puede ser pintura.

La pregunta que fue el punto de partida de todo lo que se ha hecho 
fue: ¿qué se puede hacer desde el lugar que ocupo? ¿Qué se puede hacer teniendo en cuenta las posibilidades y los recursos asequibles que se tienen? ¿Cómo puedo aportar mi grano de arena al campo de la cerámica en Colombia? Pensar en cambiar el campo de la cerámica en Colombia es una tarea muy grande e inalcanzable si no hay un trabajo personal de cada ceramista y sobretodo una unión entre todos los interesados en el campo. Debe existir un interés por querer mejorarla y se deben construir acciones en pro de este fin e interés común.

En ese sentido hay tres intensiones que están presentes en cada una de las actividades que he desarrollado:

1. "El cambio de mirada". A partir del cuestionamiento sobre el concepto heredado de la cerámica, se busca contrastar y cuestionar los paradigmas de la cerámica haciendo un paralelo entre la cerámica de Colombia, precolombina y contemporánea y lo que se hace en el campo, a nivel mundial. Conocer nuestra historia para así generar un sentimiento de pertenencia, aprecio y respeto por este saber-hacer propio.

2. Creación de espacios para la investigación de las técnicas, los conceptos y la historia. Las técnicas cerámicas son diversas y requieren de una exploración rigurosa y dedicada, haciéndose vital el trabajo de laboratorio y la exploración. Estos espacios pueden ser muy diversos y versátiles, buscan dar a conocer y dar herramientas técnicas, históricas y conceptuales de la cerámica. Es la oportunidad de vivenciar la experiencia de la cerámica en múltiples niveles.

3. Incentivar a los interesados a desarrollar obras cerámicas de alta calidad exigiéndoles y dándoles a conocer las posibilidades de la arcilla y referencias de escultores-ceramistas reconocidos mundialmente. Es a partir de la muestra e interacción con pares académicos o ceramistas-artistas invitados que se logra impulsar y dar fuerza a los procesos de investigacióncreación que busca posicionar a la cerámica como arte.

La cerámica es pues un lenguaje artístico y “(...) el artista ceramista, al igual que el pintor, elaboran su propia obra revelando en sus formas y colores el deseo expresivo de ir más allá de las técnicas simples (...) produciendo variaciones individuales y sutiles que invitan a la contemplación estética". (Duncan)

Estas tres intensiones dan origen a actividades y propuestas desarrolladas desde el 2013 y de las cuales se enunciaran las más relevantes. 


\section{Curso Cerámica Artística}

$\mathrm{E}$ ste curso indaga e invita a explorar la materia cerámica desde sus componentes conceptuales, técnicos, históricos y prácticos con el fin de contribuir a la creación de una experiencia y unos criterios claros de la cerámica. Plantea una inmersión en el mundo cerámico en donde esta se entiende como un todo. Este curso desarrolla sus contenidos estructurándose en tres módulos o partes. El primer módulo es un espacio de comprensión del lenguaje cerámico, a partir de la cual el estudiante comienza a despertar la percepción táctil y todo lo que esta implica. Sobre las técnicas de elaboración, la historia de las primeras culturas que hicieron cerámica y el conocimiento del espacio y los materiales cerámicos.

La mayoría de personas que se interesan y seleccionan este curso quieren reivindicarse con sus manos y el hacer. Esta genuina búsqueda de conexión entre el pensamiento y la mano es a la vez una forma de materializar las ideas, volver tangible algo que se piensa y poder sentirse satisfecho por el trabajo realizado. Como lo plantea Richard Sennett en su texto "El Artesano" es necesario el hacer, el repetir para aprender, hemos olvidado la mano ya que "(...) la tecnología (...) priva a los usuarios precisamente de ese concreto y repetitivo entrenamiento manual" (Senneth, 2009). Por medio de la mano nos apropiamos y entendemos las cosas que nos rodean, nos acercamos a los procesos y a la experiencia de las cosas. Es así como uno de los primeros ejercicios después de la visita guiada por el Taller, de escuchar su historia, la introducción a los materiales, técnicas de elaboración, hornos y acabados y la preparación de las pastas cerámicas, se elabora un cuenco al ser este una de las primeras formas contenedoras creadas. Unos de estos se hace viendo y otros sin ver. Es así como se empieza a entender el tacto, la presione, el grosor, la temperatura de la arcilla, sus estados y posteriormente a comprender la idea de serie a través de la repetición.

Es a través del tacto que llegamos a conocer sus tiempos y procesos; se involucra nuestro cuerpo y permite proyectarnos en un presente y ahora gracias a su experiencia sensorial en un mundo donde se privilegia lo visual.

Mi primer acercamiento con la arcilla no solo me permitió adquirir cierta sensibilidad y habilidad frente al material, sino que logró que el taller se convirtiera en mi lugar feliz, que fuera un espacio atemporal, donde podía pasar horas de ardua repetición y concentración manual y mental (Prieto, 2017). Estudiante 2017. 
Una vez finalizado la realización de una serie de cuencos se da un espacio de creación. Partiendo de los cuencos desarrollados cada estudiante debe crear una pieza nueva a partir de cortar, ensamblar, decorar, etc. Es así como el aprendizaje de las técnicas de elaboración están ligados a un ejercicio creativo y a la reflexión sobre la cerámica a partir de lecturas como El Artesano de Richard Senneth. Lectura que se plantea en el segundo módulo una vez ellos tenga la experiencia del hacer cerámico para que desde su experiencia puedan apropiar los conceptos que plantea Senneth en el capítulo La mano. Asi mismo, brinda conocimientos sobre acabados cerámicos, tipos de hornos y el desarrollo de estos en la historia.

Por último tenemos un módulo de cierre en el cual cada estudiante se plantea un proyecto personal como trabajo final desde el cual apropia los conocimientos adquiridos, proponiendo la elaboración de una o varias piezas siendo consciente y decidiendo el concepto, la técnica, el acabado, la cocción etc.

Esta clase tiene una intensidad horaria 6 horas semanales con una duración de 16 semanas.

\section{Curso Procesos del Fuego}

S e crea este curso desde el 2014 y retoma el nombre de otra electiva cerrada. Este curso busca repensar la cerámica como una práctica artística y plástica, reconociéndola como un hacer propio del territorio en el que habitamos, de nuestra historia, explorando sus posibilidades creativas y técnicas y entendiendo la relación existente entre su pasado, presente y futuro. Es así como esta electiva, para los estudiantes de la Universidad y optativa para los estudiantes de Artes, se plantea explorar los procesos escultóricos posibles con la cerámica y la investigación-creación en el desarrollo de una obra cerámica. Se parte de una visita al Museo Nacional de Colombia donde encontramos piezas cerámicas precolombinas y comprendiendo su historia, cosmogonía, usos, técnicas y formas se busca descontextualizar los investigado, apropiando y creando algo nuevo. Cada estudiante debe investigar sobre una pieza seleccionada del museo y desarrollar una pieza cerámica con elementos que se descontextualizan. Esto genera una valoración del hacer cerámico precolombino pero al mismo tiempo una comprensión de la construcción de pensamientos en el desarrollo artístico y de reflexión plástica. Una vez se logra transformar la mirada con la que llegan de la cerámica se busca enriquecerla y ampliarla. Este curso es un espacio de diálogo y apertura 
gracias a la implementación de sesiones temáticas en las cuales Se cuenta con la participación de invitados de los temas tratados, proyección de videos, presentaciones, quemas alternativas y visitas a exposiciones en donde el estudiante está en contacto con el medio de la cerámica. Charlas y prácticas sobre el sonido y la cerámica, la arquitectura con arcilla o Vernácula, aspectos geológicos de los materiales cerámicos, los tipos de quemas y hornos, Quema de cerámica Rakú, tipos de acabados, las formas constructivas, posibilidades artísticas y de instalación y la historia de la cerámica.

Hemos tenido de invitados a las artistas Juliana Góngora y Eulalia de Valdenebro, a los músicos Gustavo Pérez, Daniel Salazar y Hernán Vargas al geólogo Ricardo Ibáñez y a otros profesionales como Juliana Bonilla.

Estas sesiones son de gran importancia ya que amplían las miradas sobre el hacer y nos hacen pensar en otras posibilidades. Paralelo al desarrollo de las charlas y retroalimentación por parte de los invitados, los videos y demás actividades, cada estudiante debe trabajar en un proyecto personal. Este se nutre de las dinámicas de clases, de las investigaciones de los estudiantes. Es así como al finalizar las 16 semanas de clases, 6 horas a la semana, cada estudiante debe exponer sus piezas finalizadas, instaladas en el espacio a modo de una muestra artística. En ella se evidencia la riqueza y diversidad de propuestas y aproximaciones a la cerámica.

En la cerámica es muy posible encontrar un camino personal sin demasiado problema porque el fuego interviene de manera tan definitiva y los procesos son tan variados y las posibilidades tan infinitas que resulta un camino sencillo, por eso estoy yo enamorado de la cerámica. Rafael Pérez (Universidad Nacional de Colombia, 2017).

\section{PLANES DE MEJORAMIENTO EN LA UNIVERSIDAD}

T a Universidad busca mejorarse para así poder brindar una educación de calidad. Es por ello que ha implementado un Plan de Mejoramiento que busca renovar los laboratorios de la Universidad. El Taller de Cerámica fue incluido como laboratorio en el 2013 y gracias a esto ha podido recibir recursos para su adecuación y mejora. En el 2014 y 2016 adquirió nuevos equipos y herramientas necesarios para el desarrollo de las actividades académicas y el mantenimiento de equipos previamente adquiridos. Esto ha posibilitado implementar nuevas prácticas y una mejor adecuación y organización del espacio. 


\section{Cursos del Programa de Extensión de la UNIVERSIDAD NACIONAL LlaMAdo Mirando HaCia Adentro: Cerámica PreColombina.}

$\mathrm{H}$

emos estado mirando hacia el exterior por muchos años, valorando más lo que viene de otros países que lo local. Hemos heredado ese desamor por lo propio debido a la falta de conocimiento sobre lo que somos y hacemos. Es por esto que se decidió abrir un curso que diera la oportunidad de ahondar, no solo en el hacer cerámico y las formas propuestas por estas culturas, sino en su significado, cosmogonía, contexto y creencias. Desde pequeños hemos aprendido la historia del mundo pero muy pocos conocemos la nuestra. El curso se presenta como un espacio de reflexión sobre nuestro legado y lo que conocemos sobre este. Es un espacio para valorar, conocer y amar lo que tenemos, lo que somos, lo que fuimos y estas raíces que se mezclan en nuestra contemporaneidad.

Diez sesiones de 4 horas por sábado es muy poco, pero nos permite entender cuáles eran las culturas existentes, las pastas cerámicas utilizadas según su territorio, la cosmogonía, los símbolos de poder, las tradiciones y la vida cotidiana. Nos permite explorar el sonido precolombino creando varias piezas sonoras, crear máscaras rituales, contenedores, formas fitomorfas, antropomorfas, zoomorfas o antropozoomorfas, que son decoradas con técnicas utilizadas por estas culturas. Aporta a la apropiación de de nuestra herencia cultural y a dar un paso a entender nuestras culturas. Esta ha sido dictada en el II periodo del 2014 hasta el II periodo de 2016.

\section{Cursos del Programa de Extensión de la UNIVERSIDAD NACIONAL LLAMADO CERÁMICA ARTÍSTICA: TÉCNICAS Y POSIBILIDADES.}

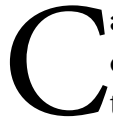
ada vez más personas se interesaban en este hacer, muchos de los estudiantes solicitaban la apertura de más espacios para el aprendizaje de técnicas y posibilidades de la cerámica. Es por esta razón que se diseña este segundo curso de extensión. Con este, se busca explorar y crear conociendo algunas de las múltiples técnicas de elaboración y acabado cerámico.

Abierto por primera vez, en el primer semestre del 2017, explora las posibilidades plásticas y de creación artística de la cerámica, entendiendo las técnicas, las características de la materia, sus requerimientos y apropiando estos conocimientos para crear una obra personal (Gracia, Laboratorio Taller de Cerámica UN de como activar y dinamizar el espacio, 2017). 
Indaga en el tipo de creaciones contemporáneas y en algunos de las técnicas de elaboración y desarrollo en los procesos de creación de ceramistas contemporáneos. Con una intensidad horaria de 4 horas cada sábado durante 10 a 11 sesiones, dependiendo del semestre, es un espacio para seguir aprendiendo sobre el inagotable mundo de la cerámica.

\section{CREACión DEL ECAC ENCUENTRO de Cerámica Artística Colombia.}

$\mathrm{E}$ 1 ECAC es un encuentro de ceramista que se creó como un espacio para reunir, difundir e intercambiar los saberes y conocimientos cerámicos para ceramistas y cualquier otra persona interesada en este lenguaje artístico. Ha buscado, desde el principio, construir un pensamiento y reflexión sobre las múltiples posibilidades del hacer para que con ello aprendamos a valorar y reconocer en la cerámica un arte.

Desde su creación en el 2013, fue pensado como un seminario que pronto creció y se convirtió en lo que conocemos hoy como un evento bienal de tipo internacional. Creado con una estructura que contempla conferencias, demostraciones, exposición, proyección de videos y una quema de Rakú. Cada una de estas actividades busca dar herramientas conceptuales, técnicas, históricas, reflexivas y críticas para entender el campo de la cerámica, poder conocer el potencial que tiene y poder enriquecer la cerámica en Colombia.

PPara la selección de las tema de las versiones y los invitados nacionales e internacionales que asisten es importante desarrollar una serie de investigaciones previas a la planificación de las versión con el fin de hacer una selección coherente de las intervenciones y participantes de las actividades. Todo teniendo en cuenta la temática de cada año. Todo esto para darle un lugar digno a la cerámica colombiana haciendo visible a ceramistas que han trabajado por años en el olvido, o silenciosamente y cuyo hacer es importante para sus regiones. Esta construcción de sentido es parte fundamental para este evento.

Cuando se mira y se conoce de primera mano la manera en la que trabajan, como operan y desarrollan su propia obra los artistas, esta es una de las mejores experiencias, la mejor clase posible. María Teresa Pardo / Coocreadora del ECAC (Universidad Nacional de Colombia, 2017). 


\section{ECAC 2014}

T a primera versión tuvo lugar en Bogotá los días 16,17 y 18 de octubre. A este se decidió invitar artistas ceramistas latinoamericanos que pudieran com1 partir sus conocimientos y su experiencia en la escena internacional. Este primer evento se llevó acabo durante el mes del arte en Bogotá, octubre, buscando posicionarse como un evento artístico dentro de los múltiples que se desarrollan durante este mes. Se abría así un nuevo espacio para la cerámica, para difundir, dar a conocer y repensar el hacer cerámico como una práctica artística múltiple. Organizado por la Universidad Nacional de Colombia el primer Encuentro conto con varios invitados ceramistas colombianos y ceramistas internacionales como Gustavo Pérez (México), Norma Grinberg y Rosana Bortolin (Brasil).

\section{DEMOSTARACIONES}

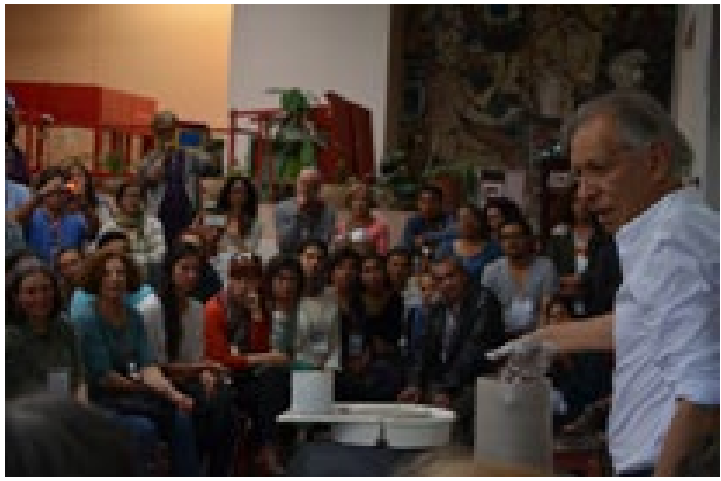

Demostración de Gustavo Perez

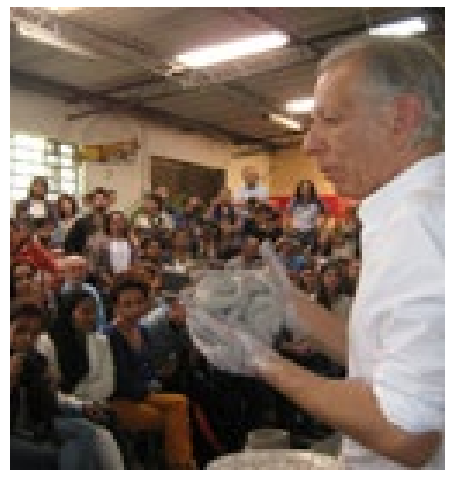

Demostración de Gustavo Perez

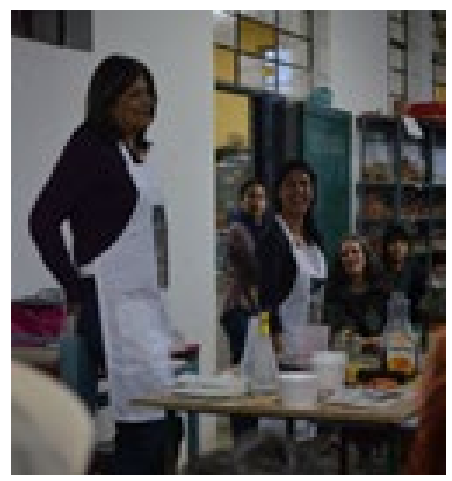

Demostración de Diana Lamir y Diana Vera 


\section{CONFERENCIAS}

Rosana Bortolin. Brasil. Nido, Casa y Cuerpo. ECA/USP Brasil.

Gustavo Pérez. México. Obra y Recorrido.

Norma Grinberg. Brasil. Recorrido, Investigación, Producción y Enseñanza.

José Ignacio Vélez. Colombia. Aportes y movimiento. Cerámica en Antioquia.

Lina Pardo. Colombia. Ser ceramista en Colombia.

Juan Camilo Chávez. Colombia. Proyecto DEL BARRO.

Ana María Lozano. Colombia. Practicas contemporáneas en la Cerámica.

\section{DEMOSTRACIONES}

Gustavo Pérez. México. Demostración Obra y Recorrido.

Norma Grinberg. Brasil. Demostración Obra y Recorrido.

Lina Pardo. Colombia. Demostración Construcción Paisajes.

Diana Lamir y Diana Vera. Colombia. Demostración Grabado y Cerámica.

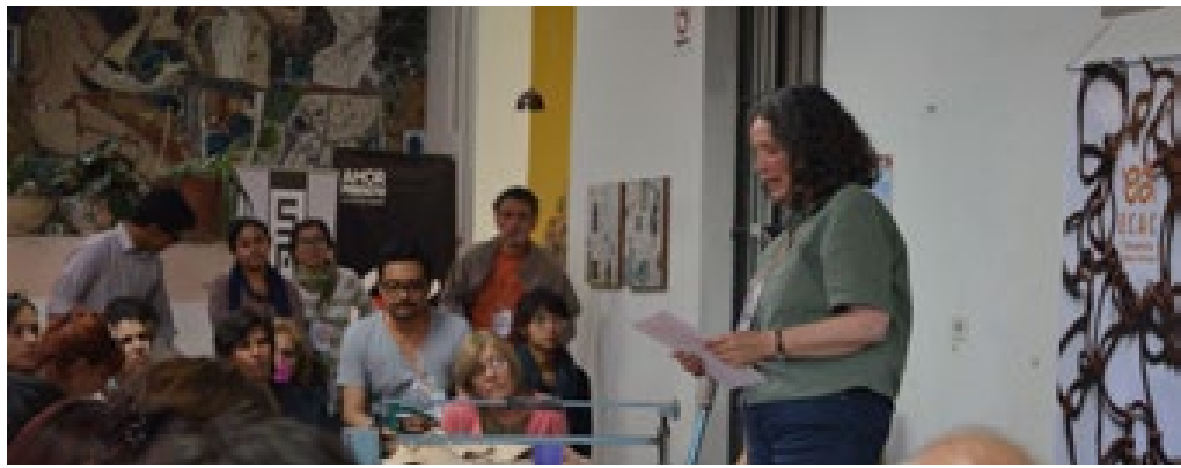

María Jerez. Colombia. Demostración Las Otilias.

Marcela Woolcott. Colombia. Demostración Acabados Cerámicos. 


\section{QUEMA DE RAKÚ}

Es un espacio lúdico de participación de todo el público; desde los ceramistas más experimentados hasta las personas que no habían tenido ninguna experiencia con la cerámica.

\section{PROYECCIÓN DE VIDEOS SOBRE LA CERÁMICA}

Las proyecciones se hicieron en el Claustro de San Agustín y los videos que se proyectaron fueron:

-Video de Enric MESTRE, Escultor-ceramista. Realizado por Javier DIEZ. - Video de Arcadi BLASCO, Escultor-ceramista. Realizado por Javier DIEZ. -Video Unto thy land. Realizado por Silvana LANDSMANN. 60 MIN. - Video Ben Nafa Ka Tia. Realizado por Jeanne DELAFOSSE. $39 \mathrm{~min}$. - Video Tierra Brillante. Realizado por José Luis FIGUEROA. 93 min.

\section{EXPOSICIÓN}

Recibe el nombre de "Cerámica Artística y su Legado" y se realizó en el Claustro de San Agustín. Sus expositores fueron: Cecilia Ordoñez, Carol Young, Dalita Navarro, Tina Vallejo, Andrea Echeverri, Cristobal Schlenker, Diana Lamir, Marcela Woolcott, Rosa María Jeréz, Andrés Sicard y Gustavo Pérez.

\section{ECAC 2016}

n su segunda versión de 2016 desarrollada los días 17, 18 y 19 de noviembre Se contó con la participación de la Embajada de Japón como invitada y con loss invitados internacionales: Chikako Yoshikawa (Japón), Kasuko Uga (Japón) y con Ruth Krauskof (Chile), Martha Pachón (Italia-Colombia), Rafael Pérez (España) y Ruth Weigand (Alemania).Estos realizaron una conferencia y nos mostraron sus técnicas y forma de hacer su obra teniendo en cuenta que la temática seleccionada para esta versión fue la Escultura Cerámica. Muchos ceramistas colombianos mostraron su obra cerámica en la exposición que se realizó en las instalaciones de la Facultad de Artes de la Pontificia Universidad Javeriana donde se encontraban propuestas y diferentes generaciones. Así mismo el espacio de Proyección de Videos conto con los videos aportados por Martha Pachón - sobre Des 
Primtemps de Potiers un evento anual que reune a los ceramistas franceses en varias actividades en la primavera, Ruth Krauskopf trajo videos sobre su obra y algunos ceramistas en Chile. Se contó con una muestra representativa de imágenes del ICANH de cerámica precolombina y un proyecto de reconocimiento de los centros cerámicos tradicionales de Colombia creados por el Ministerio de Cultura. Este proyecto publicado en formato de cuadernos y videos fue llamado "Cuadernos de Barro".

\section{CONFERENCIAS}

RAFAEL PÉREZ - España; "Obra y trayectoria”.

CHIKAKO YOSHIKAWA - Japón; "Hornos tradicionales Japoneses" RUTH WEIGAND- Alemania; '/Movimiento/ Espacio y Forma'/

RUTH KRAUSKOPF - Chile "Obra y aportes a la Cerámica en Chile" KASUKO UGA- Japón 'Tokoname”.

MARTHA PACHÓN - Colombia-Italia /Una obra polivalente de América a Europa.

\section{DEMOSTRACIONES}

Las demostraciones desarrolladas por cada uno de los invitados daban la posibilidad de visibilizar algunos de sus procesos de creación. Evidenciaban su maestría y técnica.

RAFAEL PÉREZ - España: Demostración Obra y Recorrido.

CHIKAKO YOSHIKAWA - Japón: Demostración Obra y Recorrido.

KASUKO UGA - Japón: Tokoname Demostración Obra y Recorrido.

RUTH KRAUSKOPF - Chile: Demostración Obra y Recorrido.

MARTHA PACHÓN - Colombia-Italia: Demostración Obra y Recorrido.

\section{QUEMA DE RAKÚ}

Para esta segunda versión se quiso contar nuevamente con la quema de Rakú al ser esta una dinámica muy festiva y que genera mucho asombro en el Publico, al evidenciar de una forma muy visible la trasformación en la cerámica. 


\section{PROYECCIÓN DE VIDEOS SOBRE LA CERÁMICA}

Las proyecciones se hicieron al mismo tiempo que se desarrollaba la quema de cerámica Rakú mientras las piezas que quemaban en el horno y las personas esperaban a que estas salieran. Los videos proyectaron en esta oportunidad fueron:

-"Cuadernos de Barro" Ministerio de Cultura - Colombia /la Chamba/ Carmen de Viboral y Raquira.

-Le Printemps de Potiers- Bandol 2015 - Francia.

-Sonidos de América de Esteban Valdivia - Argentina.

-Colección de cerámica prehispánica - Instituto Colombiano de Antropología e Historia ICANH. Nicolás Bonilla Maldonado.

-Entrevista de Marilú Rosenthal a Ruth Krauskopf.

-Shozo Michikawa - El artista construye una escultura al ritmo de música prehispánica, Santiago Chile.

\section{EXPOSICIÓN}

Para el Segundo Encuentro de Cerámica Artística, se abre una convocatoria para las personas interesadas en mostrar su trabajo en la Exposición ECAC 2016 a diferencia del Primer ECAC, en donde se invitaron personalmente a los artistas-ceramistas relacionados con el arte y la educación en la Universidad Nacional y otros que habían aportado al campo.

Actualmente se está organizando el ECAC 2018 que tendrá lugar en la segunda mitad del año los días 1,2 y 3 de noviembre.

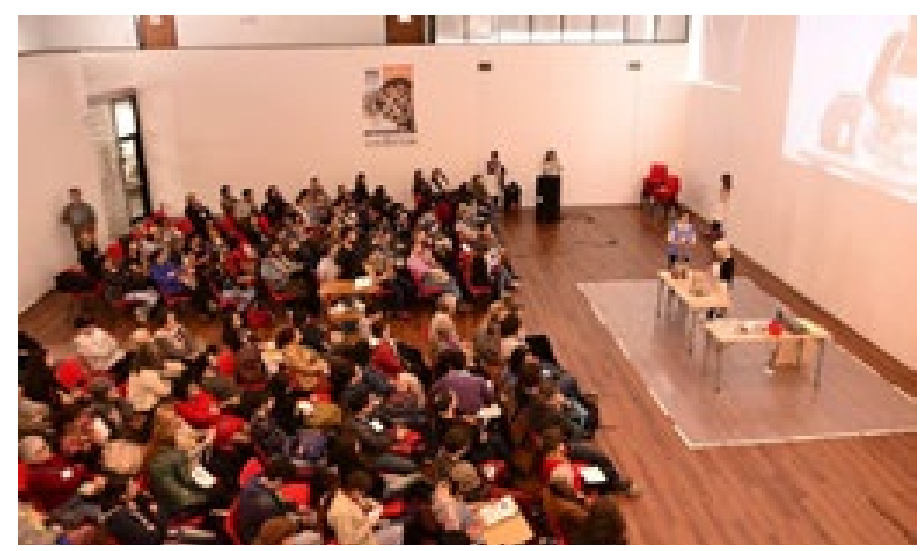

Vista general del espacio durante las demonstraciones 


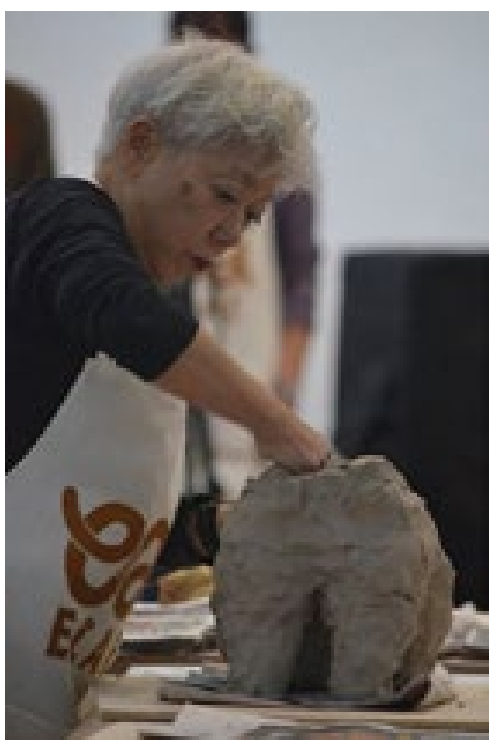

Demostración Chikako Yosikawa
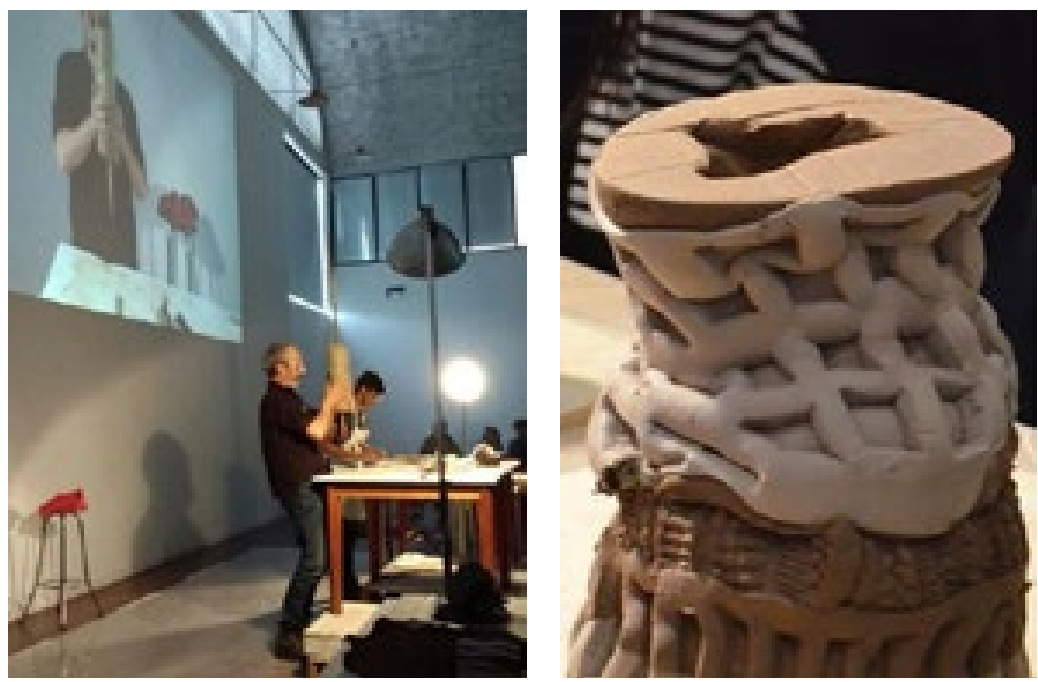

Demostración Rafael Pérez 


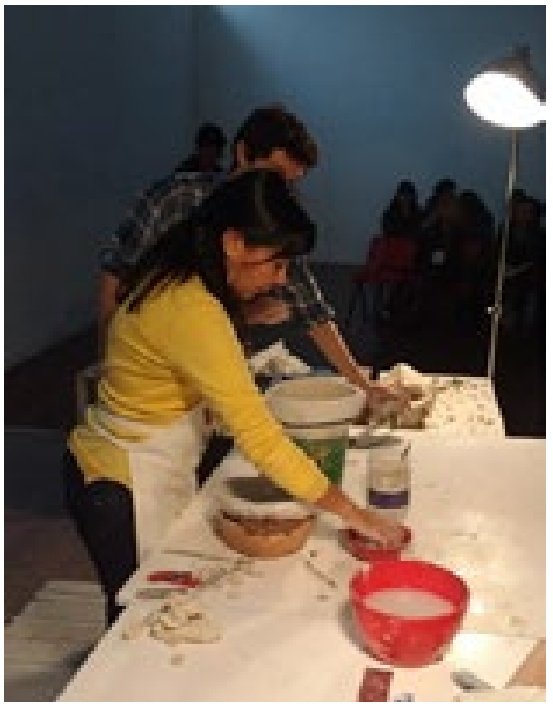

Demostración Martha Pachón

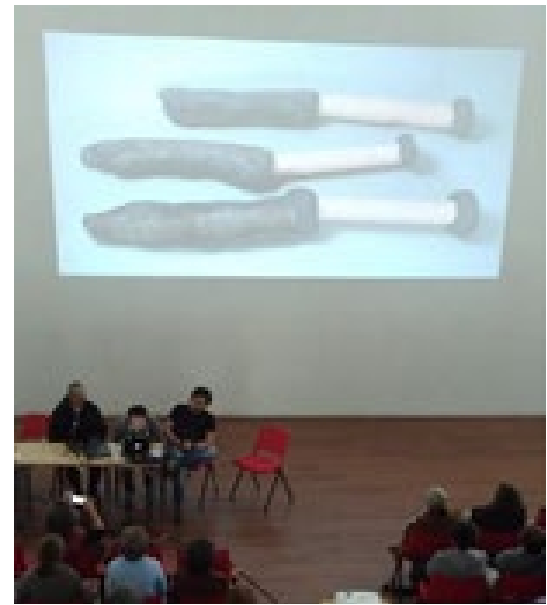

Conferencia Rafael Pérez

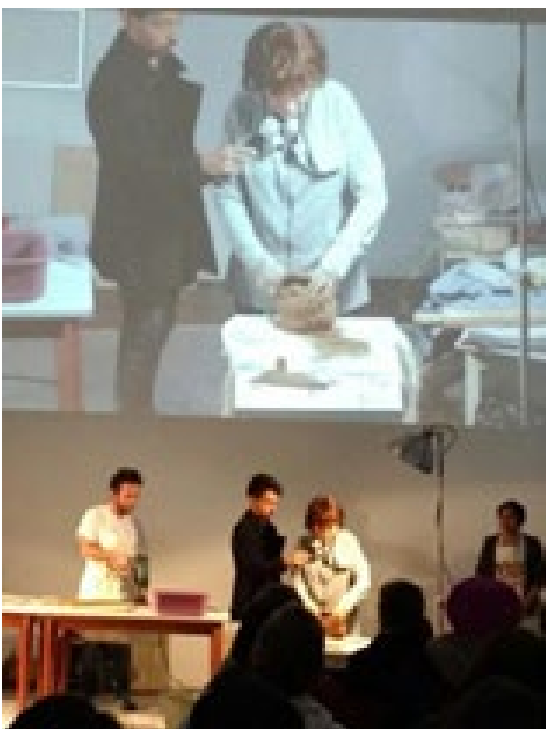

Demostración Ruth Krauskopf

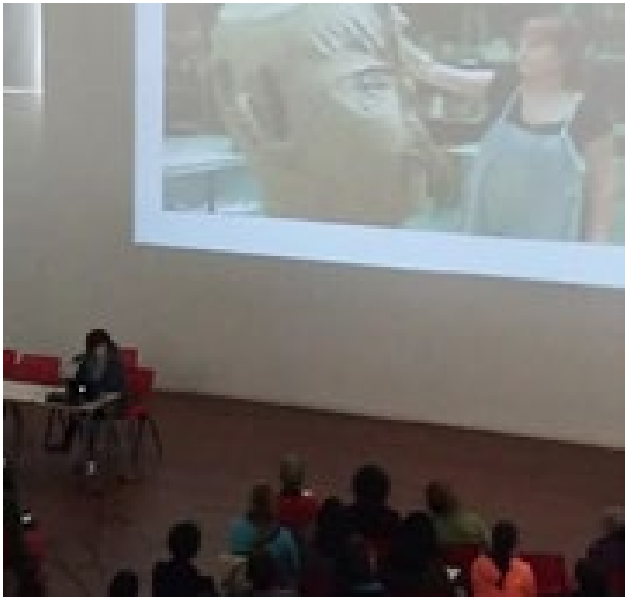

Conferencia Ruth Krauskopf 

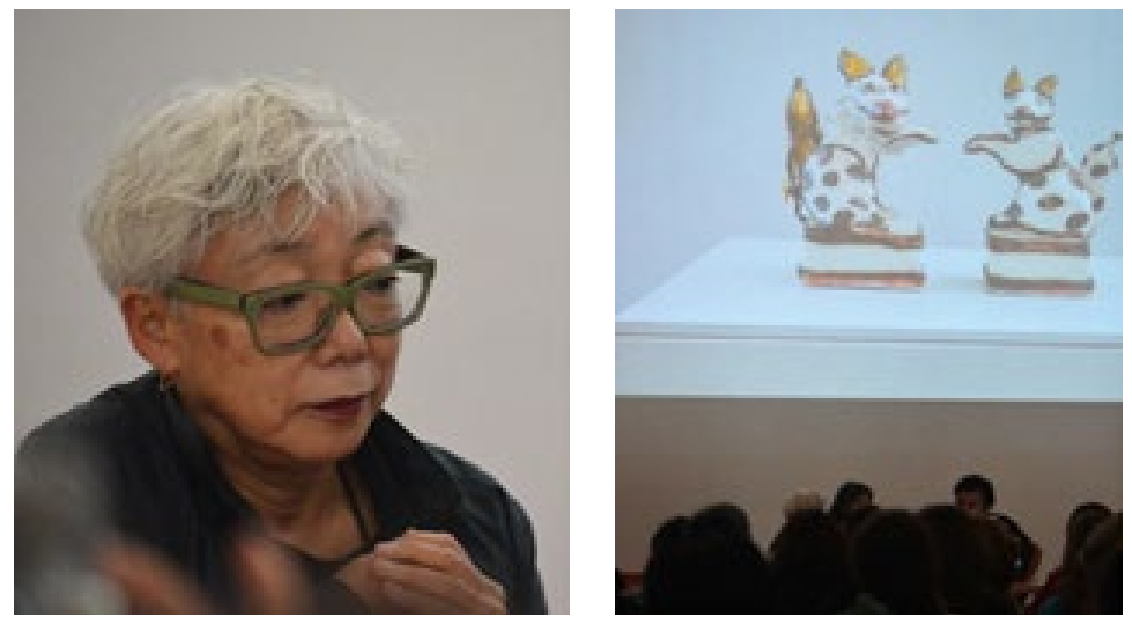

Conferencia Chikako Yoshikawa

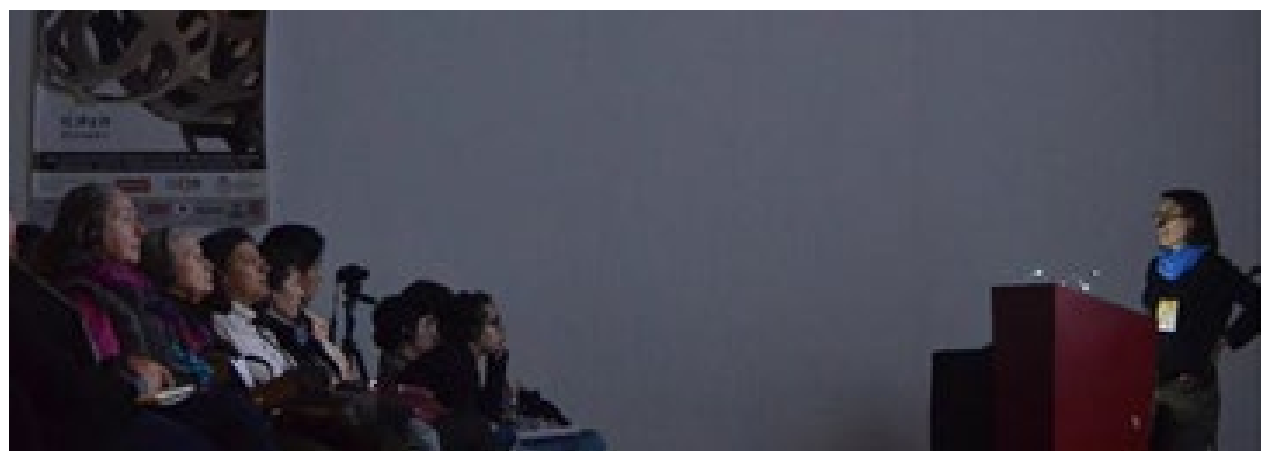

Conferencia Ruth Weigand 

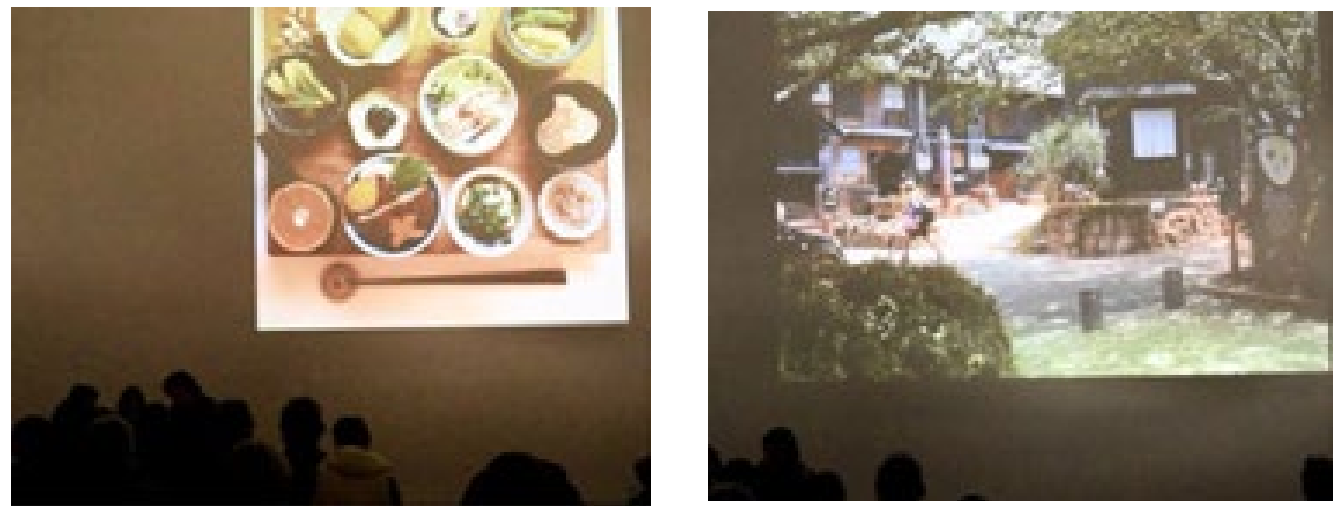

Conferencia KASUKO UGA
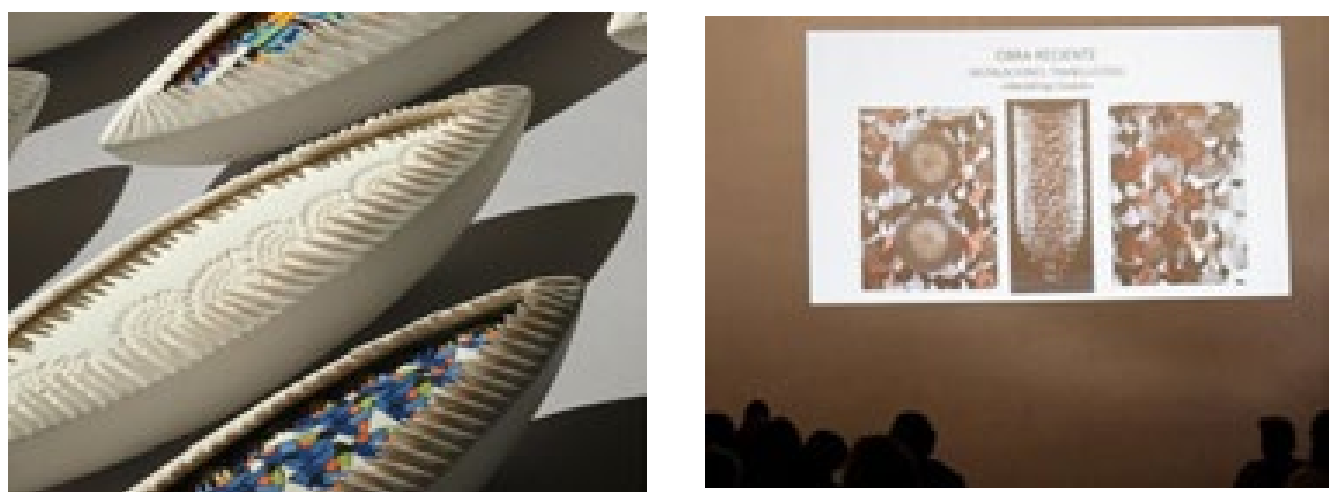

Conferencia MARTHA PACHÓN 

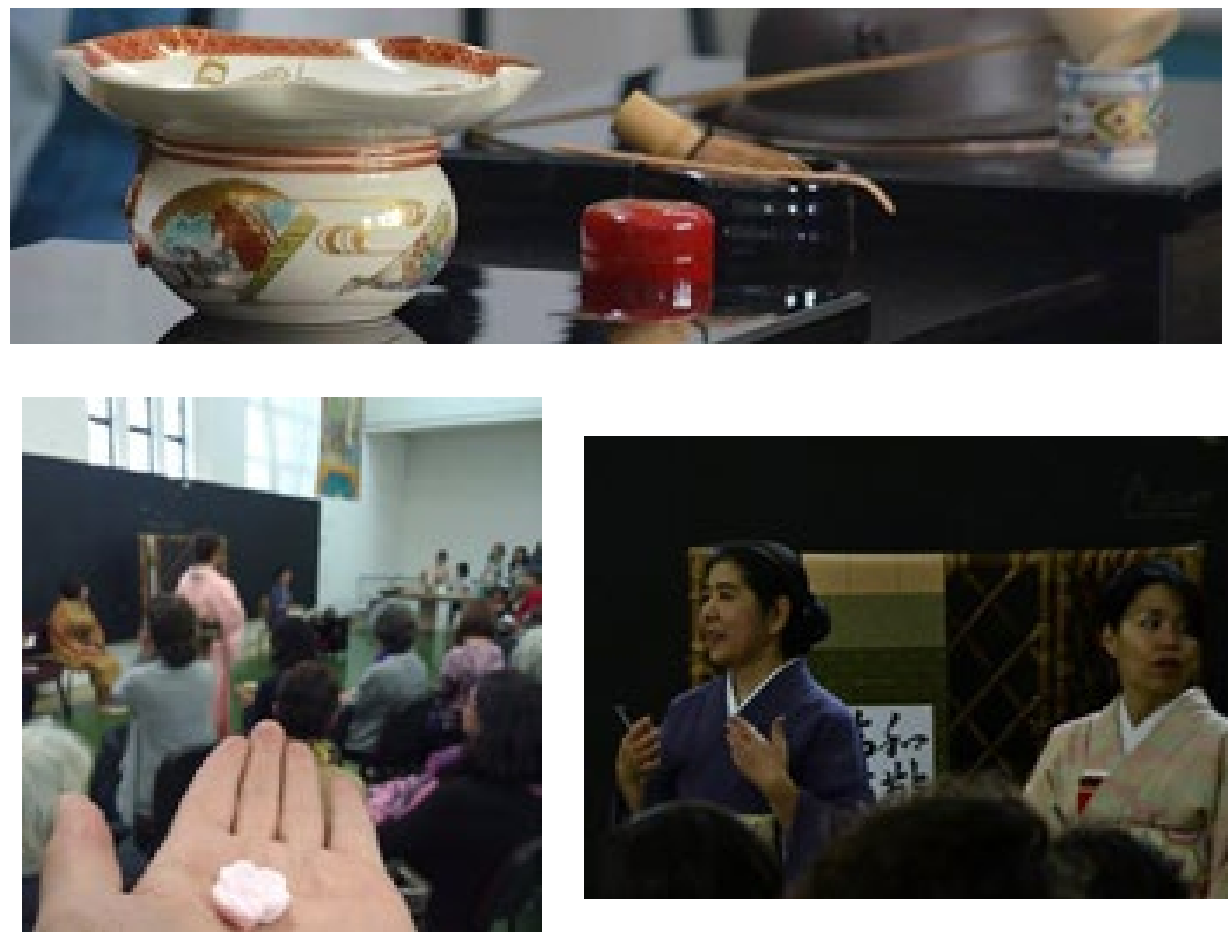

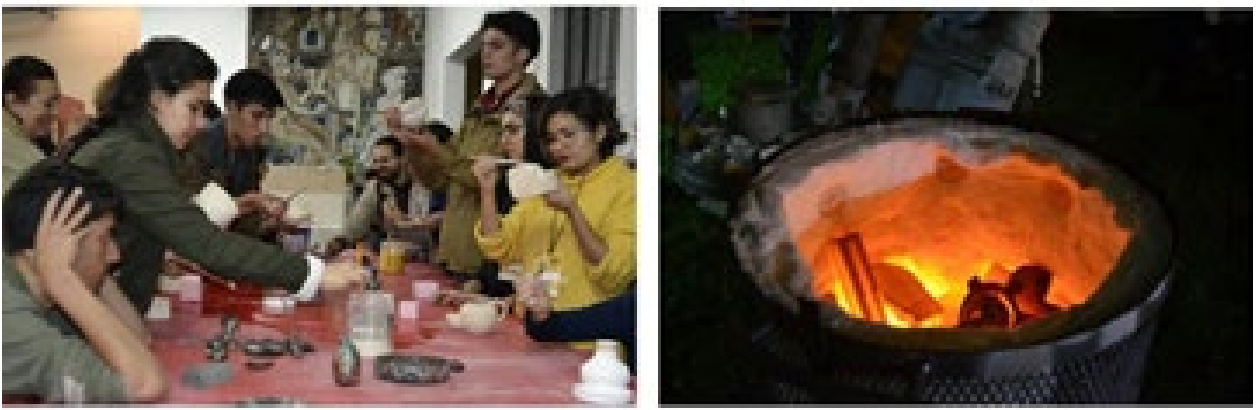

PROYECCIÓN DE VIDEOS SOBRE LA CERÁMICA
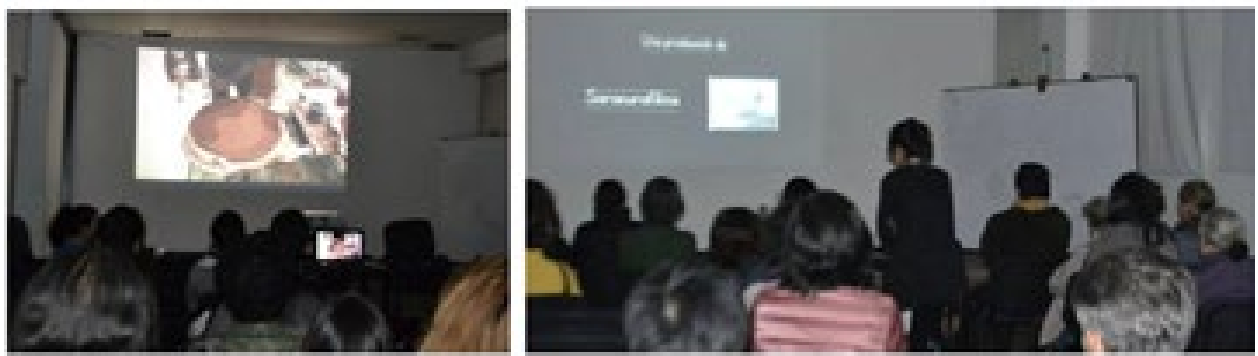


\section{CONFERENCIAS}
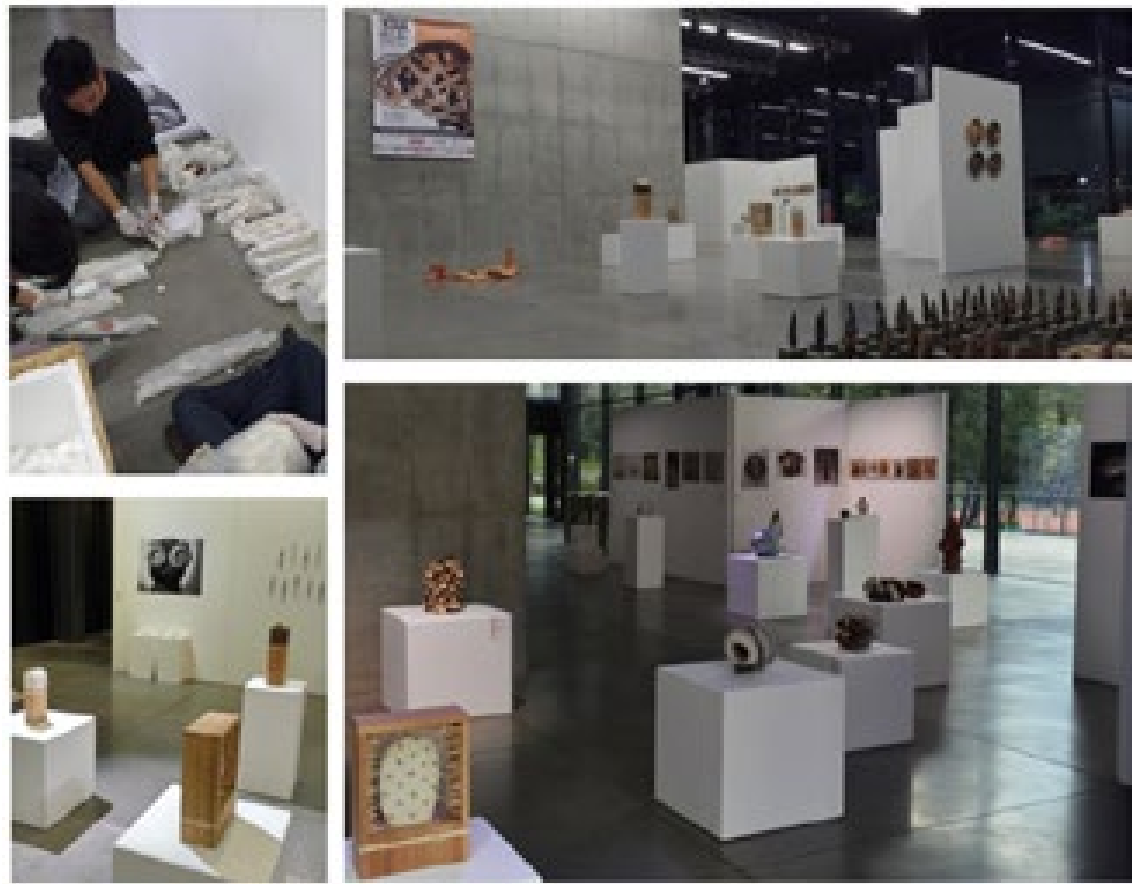

El ECAC se presenta como una experiencia enriquecedora que viene a llenar un vacío presente en nuestro medio y busca suscitar y generar nuevas propuesta para el campo de la cerámica. Esta creo vínculos desde las prácticas cerámicas y posibilitó la cooperación y el trabajo interinstitucional entre estudiantes de diversas universidades y diferentes generaciones de ceramistas. Esta iniciativa nos ha demandado mucho esfuerzo y dedicación pero sabemos es un espacio apropiado para contribuir al mejoramiento del campo de la cerámica para poder llegar a tener un futuro lleno de propuesta con una cerámica revitalizada.

Link ECAC: http://www.facartes.unal.edu.co/ecac/ 


\section{COMENTARIOS DE LOS ASISTENTES DEL ECAC 2014 Y 2016:}

Este evento que surgió como una pequeña iniciativa haido creciendo gracias a las buenas ideas y todo el grupo de trabajo que durante años ha estado pendiente de todos los requerimientos y logística que un evento de este tipo requiere. En estos dos años hemos escuchado comentarios que no hacen otra cosa que motivarnos a continuar. A continuación compartimos algunos de estos comentarios.

1. Este primer encuentro de cerámica artística ha sido una gran sorpresa. Todo llega a su tiempo, pero es un primer gran paso, para de la mano seguir caminando con diferentes direcciones que nos unan. Aprecio mucho el esfuerzo y el trabajo de los organizadores y de todas las personas detrás de este gran evento y la generosidad de todos los invitados, muchas gracias!. Pero, sin embargo, tendría que haber otra estrategia para que el evento se dé a conocer y sea más masivo. Yo sé que nos conocemos muy poco entre los ceramistas aquí en Bogotá y en Colombia, por lo tanto valoro mucho este encuentro, los diálogos, las preguntas y nuevamente la generosidad de todos los que amablemente vinieron a compartir información pertinente a su obra y al oficio. Mil gracias. Lina Caro

2. Este encuentro ha sido la oportunidad para aprender de la generosidad de todos y cada uno de los participantes. Gracias por su generosidad y que sea ésta la puerta abierta para nuevas experiencias. Agradezco desde ya que me tengan en cuenta para todos los encuentros de experiencias artísticas. El equipo de este encuentro es de una gran calidad humana. Muchas Gracias y un abrazo grande. Martha Uribe

3. Primer Encuentro de Cerámica Artística. Debo agradecer la oportunidad que han brindado de compartir y aprender cerca de la cerámica con el encuentro de varios maestros y saberes distintos; la interacción con los otros y tener un interés en común. Desearía que fuera el primero de muchos espacios para consolidar y validar la cerámica como una práctica necesaria para la expresión del ser. Kimberly Ortiz Galeano.

4. El evento ha sido un éxito desde mi percepción. A nivel de aprendizaje ha sido muy significativo, todos los ponentes invitados han proporcionado información y anécdotas que resultan valiosas al estar comenzando en este proceso, en este trabajo. La actividad de demostraciones en el taller fue un acto bello donde hemos podido apreciar 
desde el hacer todas las experiencias de los artistas invitados. Gracias por la organización de este evento. Espero que se vuelva a dar otra versión y en esa versión deseo ayudar activamente. Gracias. María Alejandra Rojas.

5. Mi gratitud es hacia ustedes, quienes nos reglaron un gratificante encuentro con la cerámica y los artistas; sin duda de gran importancia en nuestro desarrollo personal y profesional. Excelentes el nivel y calidad de los conferencistas, la programación y asistencia; mil gracias en especial por su esmerada atención. Espero poder participar en el segundo encuentro. Yaneth Ordoñez

6. Excelente encuentro, una gran oportunidad para el crecimiento de los artistas de la cerámica y de la comunidad. Muy bien escogidos los artista internacionales. Evento de una calidad muy alta que merece seguir creciendo y consolidándose como un encuentro que pueda atraer incluso artistas de la cerámica de otros países. Buen trabajo, una felicitación a todas las personas que tuvieron parte en la organización y en hacer posible este encuentro.

La cerámica ha sido un lenguaje muy recurrente en la obra de varios artistas. Desde hace algunos años los artistas han utilizado la arcilla quemada con fines artísticos diferentes hablando de "(...) diversidad de sus propuestas, su pertinencia y su fuerza, poniendo en evidencia el desplazamiento de la práctica de ceramista de la forma y contenido. Sus instalaciones, performance, esculturas y construcciones exploran la historia, el tiempo, el espacio, el plano, el color, la superficie, la relación de unos con otros, requieren de la reflexión y no simplemente algo aprendido en el instante" (Art Press 2, 2013, pág. 30) Exploran en la cerámica por la diversidad con la que cada uno puede expresarse en esta. El lenguaje cerámico está por descubrir, por explorar y para ayudar a que esto suceda se debe propiciar la experimentación colectiva e individual en la investigación creación, en donde las personas puede conocer y vivenciar el lenguaje cerámico desde todos los espacios y lugares. Es por ello que es importante visibilizar el hacer cerámico para cada vez más personas lo conozcan y quieran vincularse a este y así puedan haber más espacios, mas muestras, mas proveedores, mas lugares de formación y podamos tener un campo de la cerámica dinámico y diverso, contribuyendo a la construcción de un conocimiento cerámico desde el arte.

Cada una de estas acciones que se han implementado y elaborado en torno a la cerámica, buscan mejorar el campo, los espacios de formación académica y pretenden llegar al desarrollo del arte cerámico en nuestro territorio. 


\section{REFERÊNCIAS}

Art Press 2. (2013). La céramique au-delà de la céramique. Art Press 2, 28-33.

Ceramique Contemporaine Biennale Internationale Vallauris 2008. (2008). Vallauris Francia: Somogy Editions d’Art.

DUNCAN, G. (s.f.). Catalogo “America y Fuego”. Galeria de Arte Deimos. GARCIA, C. L. (13 de noviembre de 2017). quadernsdigitals.net. Obtenido de Signos. Teoría y práctica de la educación, 8/9 Enero Junio de 1993. Páginas 84/93 ISSN: 1131-8600: http://www.quadernsdigitals.net/index. php?accionMenu=hemeroteca.VisualizaArticuloIU.visualiza\&articulo_ $\mathrm{id}=601$

GRACIA Alcazar, D. E., \& Pardo, M. T. (2014). 1er Encuentro de Cerámica Artística Colombia ECAC 2014. Bogota.

GRACIA, D. E. (2009). Volumen 2 Reflexiones. Escuela de Artes Plasticas Universidad Nacional. Bogotá: Trabajo de grado.

GRACIA, D. E. (2017). Laboratorio Taller de Cerámica UN de como activar y dinamizar el espacio. (U. N. Colombia, Ed.) Ars 301 revista, 2, 106-109.

PARDO, M. T., \& Gracia Alcazar, D. E. (2017). 2do Encuentro de Cerámica Artistica Colombia ECAC 2016. Bogotá.

POTIERS, L. P. (Dirección). (2015). Rencontres techniques et Trasmission 2015 [Película].

PRIETO, C. J. (25 de septiembre de 2017). Mi experiecia en el Taller / texto de clase. Bogota, Colombia.

SENNETH, R. (2009). El Artesano. Barcelona: Anagrama.

The Garth Clarck and Mark del Vecchio Collection. (2014). Art Opening: Shifting paradigms in contemporary Ceramics. The Garth Clarck and Mark del Vecchio.

TRABA, M. ( 1985). Historia abierta del Arte Colombiano. Instituto Colombiano de Cultura.

Universidad Nacional de Colombia. (27 de 10 de 2017). https://www. youtube.com/watch?v=YjzbfCz-QxI. Obtenido de https://www.youtube. $\mathrm{com} /$ watch? $\mathrm{v}=\mathrm{YjzbfCz}-\mathrm{QxI}$ 